\title{
Superradiance and anomalous hyperfine splitting in inhomogeneous ensembles
}

\author{
Petar Andrejić $\circledast^{1, *}$ and Adriana Pálffy ${ }^{1,2, *}$ \\ ${ }^{1}$ Max Planck Institut für Kernphysik, Saupfercheckweg 1, 69117 Heidelberg, Germany \\ ${ }^{2}$ Department of Physics, Friedrich-Alexander-Universität Erlangen-Nürnberg, 91058 Erlangen, Germany
}

(Received 24 February 2021; accepted 2 August 2021; published 7 September 2021)

\begin{abstract}
Collective effects in the interaction of light with ensembles of identical scatterers play an important role in many fields of physics. However, often the term "identical" is not accurate due to the presence of hyperfine fields which induce inhomogeneous transition shifts and splittings. Here we develop a formalism based on the Green'sfunction method to model the linear response of such inhomogeneous ensembles in one-dimensional waveguides. We obtain a compact formula for the collective spectrum, which exhibits deviations from the uniform frequency shift and broadening expected of two-level systems. In particular, if the coherent contribution to the collective coupling is large, the effect of inhomogeneous broadening can be suppressed, with the linewidth approaching that of the superradiant value. We apply this formalism to describe collective effects in x-ray scattering off thin-film waveguides for inhomogeneous hyperfine parameters.
\end{abstract}

DOI: 10.1103/PhysRevA.104.033702

\section{INTRODUCTION}

When an ensemble of identical atoms interacts with light of wavelength much larger than the size of the ensemble, the atoms absorb and emit radiation collectively, resulting in the phenomenon of superradiance. This was first investigated theoretically by Dicke for the case of two-level systems in [1]. Provided the average interparticle distance is much smaller than the average radiated wavelength, the permutational invariance of the collective light-matter interaction results in the $N$ atoms of the ensemble absorbing and emitting radiation collectively, with a factor of $N$ enhancement of the decay rate compared to the single-atom value [1-4]. Following the pioneering experiment by Skribanowitz et al. [5], superradiance has been demonstrated and studied in many systems, such as Bose-Einstein condensates [6,7], quantum dots [8], color centers [9], cold atoms [10,11], Mössbauer nuclei [12-14], and trapped atoms coupled to a cavity $[15,16]$, to name a few. Thus, the concept of superradiance has implications in many fields, such as quantum information [17,18], cavity quantum electrodynamics [15,16], astrophysics [19], and advanced light sources [20].

The mechanism behind superradiance and collective emission also applies to multilevel systems, and extensions to the Dicke model for multilevel atoms have been explored [21-27]. Like the Dicke model, these assume completely

\footnotetext{
*petar.andrejic@mpi-hd.mpg.de

†adriana.palffy-buss@fau.de
}

Published by the American Physical Society under the terms of the Creative Commons Attribution 4.0 International license. Further distribution of this work must maintain attribution to the author(s) and the published article's title, journal citation, and DOI. Open access publication funded by the Max Planck Society. uniform illumination of the ensemble of atoms and that the latter are all completely identical. In parallel, since the 1970s theoretical works have been addressing the effect of inhomogeneous broadening on superradiance [28-38] and radiation trapping [39]. This is pertinent to many physical systems where the energy levels and decay rates are different due to local environment effects such as Zeeman and hyperfine splittings induced by magnetic or electric fields, Doppler broadening due to thermal interactions, or simply fabrication in the case of quantum dots. Inhomogeneous broadening effects have been investigated also in the related process of superfluorescence [40-42]. It is the purpose of this work to present a versatile formalism which allows us to theoretically model the superradiant response of multilevel systems in onedimensional waveguides, where at the same time the emitters are inhomogeneous.

One-dimensional waveguides play a special role in superradiance because they facilitate the otherwise challenging uniform illumination of the scatterer ensemble. Superradiance relies on permutation invariance, i.e., the invariance of the system under the exchange of any two scatterers in the ensemble, which in turn relies on uniform illumination of the ensemble. In the single-photon regime, incident pulses are reemitted in a highly directional manner $[10,17]$, preserving the incident wave vector. As such, it has been found that arranging the atoms in quasi-one-dimensional arrangements such as pencil geometries can enhance superradiance [43]. Also, uniform illumination can be easily achieved if the atoms are placed in a one-dimensional waveguidelike structure [44-46] or from x-ray grazing incidence reflection from thin films [47-51]. The atomic excitations propagate through the waveguide as a polariton and the waveguide structure restricts the propagation of the scattered light to onedimensional plane-wave propagation. This results in uniform illumination, with translational symmetry playing the role of permutational symmetry, achieving superradiance without 
requiring the wavelength to be much larger than the atomic spacing.

In this work we investigate the superradiant response of multilevel and inhomogeneous scatterers, e.g., atoms or nuclei, in one-dimensional waveguides. If the length scale of the environmental variation is much larger than the emitter spacing, the ensemble can be partitioned into approximately uniform subensembles. Thus, the collective interaction can still play a significant role; however, the interplay between the spectral inhomogeneity and the collective scattering results in nontrivial structure of the resulting spectra. To describe the dipole-dipole interaction we use a Green's-function method, developed by Welsch and co-workers [52,53], which has been successfully applied to describe superradiance in diverse systems such as atomic clouds $[10,11,54,55]$, one-dimensional waveguides $[44,45]$, and thin-film x-ray reflection [51,56]. A related multiple-scattering formulation by Wubs et al. [57] also relates the scattered response of resonant atoms embedded in a linear dielectric medium to a Lippmann-Schwinger equation involving the Green's function of the medium.

Using the Green's-function approach, a compact formula is found for the weak-excitation regime susceptibility, in terms of the coherent average of the emitters responses, with the collective interaction describable via a single complex constant. This allows the effects of inhomogeneities and of the collective interaction to be analyzed separately, allowing for a better understanding of their respective contributions to the collective spectrum.

We apply this formalism to the concrete example of $\mathrm{x}$-ray quantum optics systems that comprise ensembles of Mössbauer nuclei in thin-film x-ray cavities. The latter are a particularly suitable platform for exploring superradiance and collective interaction between emitters. In these systems, a thin layer of resonant nuclei is placed in the center of a thinfilm cavity, forming a waveguidelike structure. Evanescent guided modes of the cavity are driven at grazing incidence, coupling to Mössbauer transitions in the nuclei. This forms an exceptionally clean system, due to the incredibly narrow linewidth of Mössbauer transitions and the large energy scale of the $\mathrm{x}$ rays resulting in effectively zero thermal noise [12]. Using this setup, superradiance of single x-ray photons was experimentally demonstrated by Röhlsberger et al. [13], observing both a collective Lamb shift and up to a factor of 61 enhancement of the decay rate. Further work by Chumakov et al. at the SACLA X-ray free-electron laser has verified the scaling behavior for multiphoton excitations [14].

However, at the same time the resonant nuclear spectrum often features hyperfine interactions due to the electronic and magnetic environment. As creating large single-crystal samples is challenging, the resonant layer will in general be polycrystalline, and therefore the nuclear hyperfine environment is typically inhomogeneous. Our results consider examples of the combination of collective and inhomogeneous effects and show that in general the addition of collective interactions is more complex than a simple broadening and Lamb shift. In particular, the coherent part of the collective coupling distorts the line shape and can even be used to reduce the broadening from the sample inhomogeneity, thus providing user control over the samples linewidth. These findings are therefore very useful both for

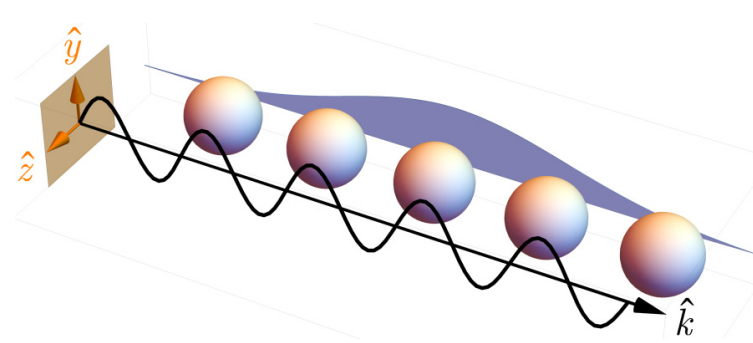

FIG. 1. Schematic plot (not to scale) of the waveguide scattering geometry. The scatterers (spheres) are lined up along the waveguide direction $\hat{k}=\hat{x}$. The restriction of propagation to this one dimension requires the polarization of the guided modes to span the $(\hat{y}, \hat{z})$ plane. As an example, we may consider a superimposed magnetic hyperfine field distribution with a Gaussian profile (blue shaded curve in the background), creating an inhomogeneity.

understanding experimental results and for designing schemes to control the collective radiation spectrum and remove unwanted features. Due to the versatility of the formalism, these results will be useful for a larger community investigating superradiant effects in various types of one-dimensional ensembles.

This work is structured as follows. Section II introduces the general model and derives the equation of motion and the collective Lamb shift and cross couplings. The application of the model to X-ray thin-film cavities with embedded layers of Mössbauer nuclei is presented in Sec. III. We put the present formalism in the context of already existing theoretical models for x-ray grazing incidence on thin-film cavities in Sec. III A. Our examples for inhomogeneous nuclear hyperfine splitting are discussed in Secs. III B and III C. Conclusions and a brief outlook are summarized in Sec. IV.

\section{MODEL}

\section{A. Hamiltonian and Lindblad operators}

We begin with an ensemble of atoms in a one-dimensional waveguide, schematically illustrated in Fig. 1. The spectral parameters of the atoms are inhomogeneous and the inhomogeneity is assumed to vary slowly over the interatomic length scale such that the atoms can be divided into equally sized subensembles that are approximately translationally and permutationally symmetric. The size of each subensemble, and hence the number required, is determined by the gradient of the inhomogeneity over the spatial extent of the atoms. The variation of the inhomogeneity across each subensemble should be taken to be small enough that it cannot be resolved within the linewidth of the transitions present and can therefore be treated as a negligible perturbation. The atoms are driven by a probe field $\mathbf{E}_{\mathrm{p}}(t) e^{i\left(k_{0} x-\omega_{0} t\right)}$ of frequency $\omega_{0}$, wave vector $k_{0}$, and uniform illumination, with a possible time-dependent envelope.

Following Refs. [45,51], we work in the rotating frame of the driving field. The internal Hamiltonian for the atoms is given by

$$
H_{\mathrm{A}}=-\sum_{n} \sum_{i \in \mathcal{D}_{n}} \sum_{\mu \in \mathcal{T}_{n}} \hbar \Delta_{\mu}\left|e_{\mu}^{(i)}\right\rangle\left\langle e_{\mu}^{(i)}\right|,
$$


where $\mathcal{D}_{n}$ is the set of atoms in subensemble $n, \mathcal{T}_{n}$ is the set of excited states of subensemble $n$, and $\Delta_{\mu} \ll \omega_{0}$ is the detuning of excited state $\mu$. Furthermore, $\hbar$ is the reduced Planck constant.

Incoherent decay is described by the Lindblad operator

$$
L_{\mathrm{A}}[\rho]=-\sum_{n} \sum_{i \in \mathcal{D}_{n}} \sum_{\mu \in \mathcal{T}_{n}} \hbar \gamma_{\mu} \mathcal{L}\left[\rho,\left|e_{\mu}^{(i)}\right\rangle\left\langle g^{(i)}|,| g^{(i)}\right\rangle\left\langle e_{\mu}^{(i)}\right|\right]
$$

where $\gamma_{\mu}$ is the natural decay rate of excited state $\mu$ and

$$
\mathcal{L}[\rho, A, B]=A B \rho+\rho A B-2 B \rho A .
$$

At low saturations, the probe field only drives dipole transitions directly accessible from the ground state $g$. It will be convenient to express the transition dipole vectors in the form $\mathbf{d}_{\mu} \wp$, with $\mathbf{d}_{\mu}$ a dimensionless vector and $\wp$ the mean dipole magnitude. The driving Hamiltonian is then given by

$$
H_{\mathrm{p}}=\wp \sum_{n} \sum_{i \in \mathcal{D}_{n}} \sum_{\mu \in \mathcal{T}_{n}} \mathbf{d}_{\mu} \cdot \mathbf{E}_{\mathrm{p}}(t) e^{i k_{0} x_{i}}\left|e_{\mu}^{(i)}\right\rangle\left\langle g^{(i)}\right|+\text { H.c. }
$$

The atoms couple collectively via a resonant dipole-dipole interaction, described by the Green's-function formalism $[45,51,52]$. In this formalism, the atoms interact via the exchange of resonant photons, which are assumed to be short lived compared with the timescale of the atomic coherences and as such can be adiabatically eliminated. A sufficient condition for this is if the Green's function for the photonic propagation is much broader in frequency space than the atomic transition width $\gamma$ [45], i.e.,

$$
\left|\omega-\omega_{0}\right|=O(\gamma) \rightarrow \overleftrightarrow{G}\left(\mathbf{r}, \mathbf{r}^{\prime}, \omega\right) \approx \overleftrightarrow{G}\left(\mathbf{r}, \mathbf{r}^{\prime}, \omega_{0}\right)
$$

where $\overleftrightarrow{G}\left(\mathbf{r}, \mathbf{r}^{\prime}, \omega_{0}\right)$ is the dyadic Green's function. This gives rise to an effective resonant dipole-dipole interaction, mediated by the short-lived scattered photons, with the matrix elements given via the classical dyadic Green's function for the waveguide,

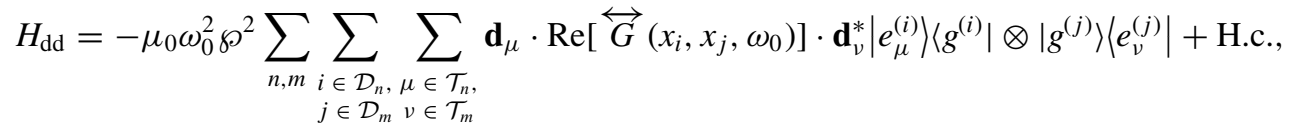

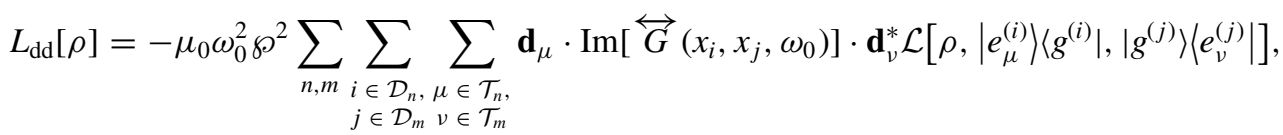

where $\overleftrightarrow{G}\left(x_{i}, x_{j}, \omega_{0}\right)$ is the dyadic Green's function for the one-dimensional waveguide and $\mu_{0}$ the vacuum permeability, respectively. Note that both coherent and incoherent scattering processes are modeled, via the Hamiltonian and Lindblad superoperators, respectively.

Due to translational symmetry, the dyadic Green's function $\overleftrightarrow{G}\left(x_{i}, x_{j}, \omega\right)$ for the waveguide can be expressed in the form

$$
\overleftrightarrow{G}\left(x_{i}, x_{j}, \omega\right)=\int \frac{d k}{2 \pi L^{-1}} \overleftrightarrow{\mathcal{G}}(k, \omega) e^{i k\left(x_{i}-x_{j}\right)}
$$

We are interested in permutationally symmetric ensembles, which require coupling to a single mode only. This can be achieved, for instance, by tailoring the ensemble positions to couple to a single eigenmode of the waveguide. Alternatively, it can also be achieved in the low-saturation regime of any ensemble that has approximately orthogonal Fourier modes, i.e., uniform illumination of ensembles with large numbers of scatterers. In this regime, the Green's-function Hamiltonian and Lindblad terms have a sharp resonance in the symmetric subspace of the driven mode (see Appendix A) and as such the collectively absorbed photons are reemitted with the same wave vector. The propagation of excitations through these coherently rescattered photons can be viewed in the Green'sfunction picture as the propagation of the atomic excitations as a polariton with well-defined momentum. At low saturations, the dynamics of the system is restricted to this polaritonic subspace. In particular, this amounts to restricting the interaction terms to the Fourier mode of the driving field,

$$
\overleftrightarrow{G}\left(x_{i}, x_{j}, \omega_{0}\right) \rightarrow \overleftrightarrow{\mathcal{G}}\left(k_{0}, \omega_{0}\right) e^{i k_{0}\left(x_{i}-x_{j}\right)}
$$

The plane-wave phase factors can then be eliminated by the following unitary transformation:

$$
\left|e_{\nu}^{(j)}\right\rangle \rightarrow e^{-i k_{0} x_{j}}\left|e_{\nu}^{(j)}\right\rangle
$$

The model then becomes permutationally symmetric. Such systems can be analyzed by a generalization of the HolsteinPrimakoff transformation [58]. This is an exact transformation that maps the collective transitions of the system to independent bosonic modes, with the collective ground state mapping to the bosonic vacuum. We introduce the bosonic creation (annihilation) operators $b_{\mu}^{\dagger}\left(b_{\mu}\right)$ for each collective transition $\mu$. For our system, the transformation reads

$$
\begin{gathered}
\sum_{i \in \mathcal{D}_{n}}\left|e_{\mu}^{(i)}\right\rangle\left\langle e_{\nu}^{(i)}\right|=b_{\mu}^{\dagger} b_{\nu}, \quad \mu, \nu \in \mathcal{T}_{n}, \\
\sum_{i \in \mathcal{D}_{n}} e^{i k_{0} x_{i}}\left|e_{\mu}^{(i)}\right\rangle\left\langle g^{(i)}\right|=b_{\mu}^{\dagger} \sqrt{N p_{n}-\sum_{\nu \in \mathcal{T}_{n}} b_{\nu}^{\dagger} b_{\nu}},
\end{gathered}
$$

where $p_{n}$ is the proportion of atoms in subensemble $n, N$ is the total number of atoms in all ensembles, and

$$
\left[b_{v}, b_{\mu}^{\dagger}\right]=\delta_{\mu \nu} \delta_{m n}, \quad \mu \in \mathcal{T}_{n}, v \in \mathcal{T}_{m}
$$

As we are interested in the linear response of our system, we have $\left\langle b_{\mu}^{\dagger} b_{\mu}\right\rangle \ll \sqrt{N}$ and we may linearize the collective transition operators

$$
\sum_{i \in \mathcal{D}_{n}} e^{i k_{0} x_{i}}\left|e_{\mu}^{(i)}\right\rangle\left\langle g^{(i)}\right| \approx \sqrt{N p_{n}} b_{\mu}^{\dagger}, \quad \mu \in \mathcal{T}_{n} .
$$


In terms of these bosonic operators, the Hamiltonian of the linearized system reads

$$
\begin{aligned}
& H_{\mathrm{A}}=-\sum_{n} \sum_{\mu \in \mathcal{T}_{n}} \hbar \Delta_{\mu} b_{\mu}^{\dagger} b_{\mu}, \\
& H_{\mathrm{dd}}=-\hbar \sum_{n, m} \sum_{\substack{\mu \in \mathcal{T}_{n}, \nu \in \mathcal{T}_{m}}} \sqrt{p_{n} p_{m}} \mathbf{d}_{\mu} \cdot \overleftrightarrow{J} \cdot \mathbf{d}_{\nu}^{*} b_{\mu}^{\dagger} b_{\nu} \\
& H_{\mathrm{p}}=\sqrt{N} \wp \sum_{n} \sum_{\mu \in \mathcal{T}_{n}} \sqrt{p_{n}} \mathbf{d}_{\mu} \cdot \mathbf{E}_{\mathrm{p}}(t) b_{\mu}^{\dagger}+\text { H.c. } \\
& L_{\mathrm{A}}[\rho]=-\sum_{n} \sum_{\mu \in \mathcal{T}_{n}} \hbar \gamma_{\mu} \mathcal{L}\left[\rho, b_{\mu}^{\dagger}, b_{\mu}\right] \\
& L_{\mathrm{dd}}[\rho]=-\hbar \Gamma \sum_{n, m} \sum_{\substack{\mu \in \mathcal{T}_{n}, \nu \in \mathcal{T}_{m}}} \sqrt{p_{n} p_{m}} \mathbf{d}_{\mu} \cdot \overleftrightarrow{\Gamma} \cdot \mathbf{d}_{\nu}^{*} \mathcal{L}\left[\rho, b_{\mu}^{\dagger}, b_{\nu}\right]
\end{aligned}
$$

where

$$
\overleftrightarrow{J}+i \overleftrightarrow{\Gamma}=N \frac{\mu_{0} \omega_{0}^{2} \wp^{2}}{\hbar} \overleftrightarrow{\mathcal{G}}\left(k_{0}, \omega_{0}\right)
$$

Note that in transforming $L_{\mathrm{A}}$ to the bosonic operators, we have assumed that the decay is dominated by the superradiance $|\Gamma| \gg \gamma_{\mu}$ such that we can approximate the single-particle decay as collective. A more exact treatment of the single-particle decay is given by Shammah et al. [59] and Gegg and Richter $[23,25]$, but it is not significant in the low-saturation regime we are considering (see Appendix B).

Finally, although we have considered only a single ground state, for systems with multiple ground states the resulting equations of motion are of the same form. The partitioning of the subensembles can be extended to partitioning by the initial ground-state configurations of the atoms. Terms that couple to a different ground-state configuration are suppressed by a factor of $\sqrt{N}$ and can be neglected (see Appendix C).

\section{B. Equation of motion}

The equation of motion for a transition operator corresponding to $\mu \in \mathcal{T}_{n}$ is given by

$$
\begin{aligned}
\partial_{t} b_{\mu}= & \frac{i}{\hbar}\left[H, b_{\mu}\right]+\frac{1}{\hbar} L\left[b_{\mu}\right] \\
= & \left(i \Delta_{\mu}-\gamma_{\mu}\right) b_{\mu} \\
& +i \sqrt{p_{n}} \mathbf{d}_{\mu} \cdot(\overleftrightarrow{J}+i \overleftrightarrow{\Gamma}) \cdot \sum_{m} \sqrt{p_{m}} \sum_{v \in T_{m}} \mathbf{d}_{v}^{*} b_{v} \\
& -i \frac{\sqrt{N} \wp}{\hbar} \sqrt{p_{n}} \mathbf{d}_{\mu} \cdot \mathbf{E}_{\mathrm{p}}(t) .
\end{aligned}
$$

Due to the one-dimensional nature of the problem, observables will depend on the dipole response only in the two-dimensional polarization space of the guided modes. As such, we need only consider the transverse polarization operator

$$
\begin{aligned}
\mathbf{P} & =\wp \sum_{n} \sum_{\mu \in \mathcal{T}_{n}} \sum_{i \in \mathcal{D}_{n}}\left|g^{(i)}\right\rangle\left\langle e_{\mu}^{(i)}\right| \mathbf{d}_{\mu} \\
& \approx \sqrt{N} \wp \sum_{n} \sqrt{p_{n}} \sum_{\mu \in \mathcal{T}_{n}} \mathbf{d}_{\mu}^{*} b_{\mu} .
\end{aligned}
$$

The susceptibility is found via solving for the linear response

$$
\mathbf{P}(\omega)=\epsilon_{0} \overleftrightarrow{\chi}(\omega) \cdot \mathbf{E}_{\mathrm{p}}(\omega)
$$

where $\epsilon_{0}$ is the vacuum permittivity. A compact solution for this can be obtained from the equation of motion in Fourier space. We first Fourier transform Eq. (15) to obtain

$$
\left(\omega+\Delta_{\mu}+i \gamma_{\mu}\right) b_{\mu}(\omega)+\sqrt{p_{n}} \mathbf{d}_{\mu} \cdot(\overleftrightarrow{J}+i \overleftrightarrow{\Gamma}) \cdot \sum_{m} \sqrt{p_{m}} \sum_{\nu \in \mathcal{T}_{m}} \mathbf{d}_{\nu}^{*} b_{\nu}(\omega)=\frac{\sqrt{N} \wp}{\hbar} \sqrt{p_{n}} \mathbf{d}_{\mu} \cdot \mathbf{E}_{\mathrm{p}}(\omega)
$$

It will be useful at this stage to define a reference frequency scale $\gamma_{0}$. For example, this could be the natural linewidth of a single atom, which for an electric dipole excitation can be expressed in terms of the mean dipole magnitude as $\omega_{0}^{3} \wp^{2}\left(3 \pi \hbar \epsilon_{0} c^{3}\right)^{-1}$.

Multiplying both sides of (18) by

$$
\frac{\sqrt{N p_{n}} \wp \mathbf{d}_{\mu}^{*}}{\omega+\Delta_{\mu}+i \gamma_{\mu}}
$$

and summing over $n, \mu \in \mathcal{T}_{n}$, we obtain

$$
\mathbf{P}(\omega)+\overleftrightarrow{\mathcal{F}}(\omega) \cdot \overleftrightarrow{G} \cdot \mathbf{P}(\omega)=\frac{N \wp^{2}}{\hbar \gamma_{0}} \overleftrightarrow{\mathcal{F}}(\omega) \cdot \mathbf{E}_{\mathrm{p}}(\omega)
$$

where $\overleftrightarrow{G}=\gamma_{0}^{-1}(\overleftrightarrow{J}+i \overleftrightarrow{\Gamma})$ and

$$
\overleftrightarrow{\mathcal{F}}(\omega)=\sum_{n} p_{n} \sum_{\mu \in \mathcal{T}_{n}} \frac{\gamma_{0} \mathbf{d}_{\mu}^{*} \otimes \mathbf{d}_{\mu}}{\omega+\Delta_{\mu}+i \gamma_{\mu}}
$$

is the layer response matrix, the coherent average of the responses of each transition in each subensemble. Note that in this context $\otimes$ refers to the outer product. Such a quantity appears in the dynamical scattering approach to x-ray propagation [see, e.g., Eq. (4.13) in [60] as well as [61]].

Solving for the polarization, we obtain

$$
\mathbf{P}(\omega)=\frac{N \wp^{2}}{\hbar \gamma_{0}}[\overleftrightarrow{\mathbb{1}}+\overleftrightarrow{\mathcal{F}}(\omega) \cdot \overleftrightarrow{G}]^{-1} \cdot \overleftrightarrow{\mathcal{F}}(\omega) \cdot \mathbf{E}_{\mathrm{p}}(\omega)
$$

This directly gives the first-order susceptibility

$$
\overleftrightarrow{\chi}(\omega)=\chi_{0}[\overleftrightarrow{\mathbb{1}}+\overleftrightarrow{\mathcal{F}}(\omega) \cdot \overleftrightarrow{G}]^{-1} \cdot \overleftrightarrow{\mathcal{F}}(\omega)
$$

where $\chi_{0}=N \wp^{2}\left(\hbar \gamma_{0} \epsilon_{0}\right)^{-1}$.

\section{Collective Lamb shift and cross couplings}

The coherent part of the collective coupling $J$ has previously been referred to as a collective Lamb shift $[13,62,63]$. Indeed, in the limit of a single uniform transition with natural 
decay width $\gamma_{0}$, the susceptibility can be treated as a scalar and is given by

$$
\chi(\omega)=\chi_{0} \frac{\gamma_{0}}{\omega+\Delta+i \gamma+J+i \Gamma},
$$

describing a single line, shifted by $J$ and broadened by $\Gamma$. However, with multilevel atoms and inhomogeneous configurations, $J$ does not act just as a Lamb shift but also provides additional cross couplings, analogous to an additional control field between transitions.

Specifically, a Lamb shift is a shift in an energy level due to emission and reabsorption of virtual photons from the same state. The analog of this in Eq. (13) is given by the diagonal matrix elements of $H_{\mathrm{dd}}$,

$$
-\hbar \mathbf{d}_{\mu} \cdot \overleftrightarrow{J} \cdot \mathbf{d}_{\mu}^{*}
$$

If the collective coupling acts purely as a Lamb shift, then each transition is simply shifted and broadened, giving a susceptibility of

$$
\overleftarrow{\chi}(\omega)=\chi_{0} \sum_{\mu} \frac{\gamma_{0} \mathbf{d}_{\mu}^{*} \otimes \mathbf{d}_{\mu}}{\omega+\Delta_{\mu}+i \gamma_{\mu}+\mathbf{d}_{\mu} \cdot \overleftrightarrow{G} \cdot \mathbf{d}_{\mu}^{*}}
$$

However, we can see in Eq. (13) that due to additional cross couplings from $J$ and spontaneously generated coherences from $\Gamma$ [64], the resulting spectrum will not be so straightforward to interpret and is more generally described by Eq. (23). Indeed, as we will see in Secs. III B and III C, the spectrum for inhomogeneous two-level atoms shows features that cannot be attributed to a simple Lamb shift. In the context of x-ray quantum optics, this was already hinted at in Ref. [64] and later on discussed in more detail for the general case in Ref. [26]. We note that, in particular, Ref. [26] has addressed the specific case of uniform magnetic hyperfine splitting, using the atomic cloud model of Svidzinsky et al. [55] to describe the collective coupling. This method derives an interaction kernel for the effective interatomic interactions, which turns out to be identical to the free-space Green's function. As such, the Hamiltonian of our model reproduces the model of Ref. [26] in the limiting case of atoms in free space and uniform magnetic splitting.

\section{APPLICATION TO X-RAY QUANTUM OPTICS}

We now discuss the application of the general model from Sec. II to X-ray quantum optics with Mössbauer nuclei and the connection to existing formalisms. The relevant experimental setup comprises so-called $\mathrm{x}$-ray thin-film cavities using grazing incidence reflection as illustrated in Fig. 2(a). In this setup, layers are stacked from alternating high- and low-atomicnumber $Z$ materials (for instance, $\mathrm{Pt}$ or Pd alternating with $\mathrm{C}$ or $\mathrm{B}_{4} \mathrm{C}$ ) to form a waveguide structure for a pulse fired at grazing incidence to the layers. A thin resonant layer of Mössbauer nuclei, for instance, ${ }^{57} \mathrm{Fe}$ or ${ }^{57} \mathrm{Fe}$-enriched stainless steel $\left({ }^{57} \mathrm{SS}\right)$, is embedded in this stack, usually sandwiched between low- $Z$ material layers. The scattering response of the system is recorded in the cavity reflectivity measured at the detector. As a function of incidence angle, the cavity reflectivity presents several minima, which correspond to the resonant driving of guided modes. An example is presented in Fig. 2(b). The minima are known as critical angles and they indicate the formation of a standing-wave structure across the cavity layers.
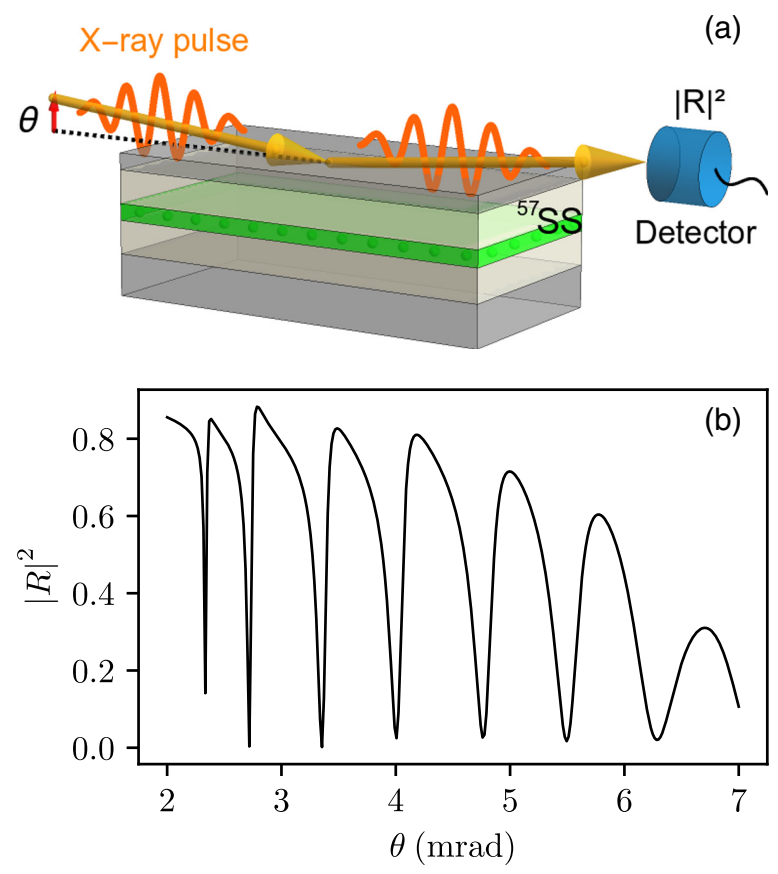

FIG. 2. (a) Schematic illustration of a thin-film cavity. $X$ rays in grazing incidence with angle $\theta$ couple evanescently to the layered structure, exciting resonant transitions in stainless steel $95 \%$ enriched with ${ }^{57} \mathrm{Fe}$ (referred to as ${ }^{57} \mathrm{SS}$ ). The cavity reflectivity $|R|^{2}$ is measured at the detector. (b) Example of theoretical reflectivity spectrum $|R|^{2}(\theta)$ for a cavity with structure $\mathrm{Pt}(2.8 \mathrm{~nm}) / \mathrm{C}(22 \mathrm{~nm}) /{ }^{57} \mathrm{SS}(0.6 \mathrm{~nm}) / \mathrm{C}(22.5 \mathrm{~nm}) / \mathrm{Pt}(15 \mathrm{~nm})$, obtained using the PYTHON library PYNUSS [65]. The reflectivity has various minima that correspond to the resonant guided modes.

The resonant layer of Mössbauer nuclei is made sufficiently thin such that the guided mode field is approximately uniform across the depth of the layer.

The calculated probe field intensity profile for the $\mathrm{Pt}(2.8 \mathrm{~nm}) / \mathrm{C}(22 \mathrm{~nm}) /{ }^{57} \mathrm{SS}(0.6 \mathrm{~nm}) / \mathrm{C}(22.5 \mathrm{~nm}) / \mathrm{Pt}(15 \mathrm{~nm})$ cavity structure and incidence angle $\theta=3.35 \mathrm{mrad}$ [corresponding to the third reflection minimum in Fig. 2(b)] is presented in Fig. 3. This example was chosen such that

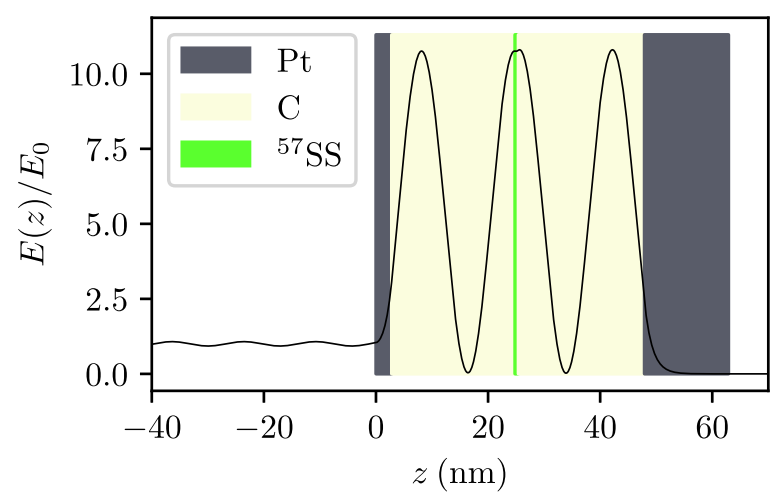

FIG. 3. Probe field intensity profile throughout the sample depth $z$ for the third reflection minimum in Fig. 2(b). The background shading illustrates the layer material, with the platinum capping layers forming the cavity and the thin layer of ${ }^{57} \mathrm{SS}$ placed at the guided mode maximum. 


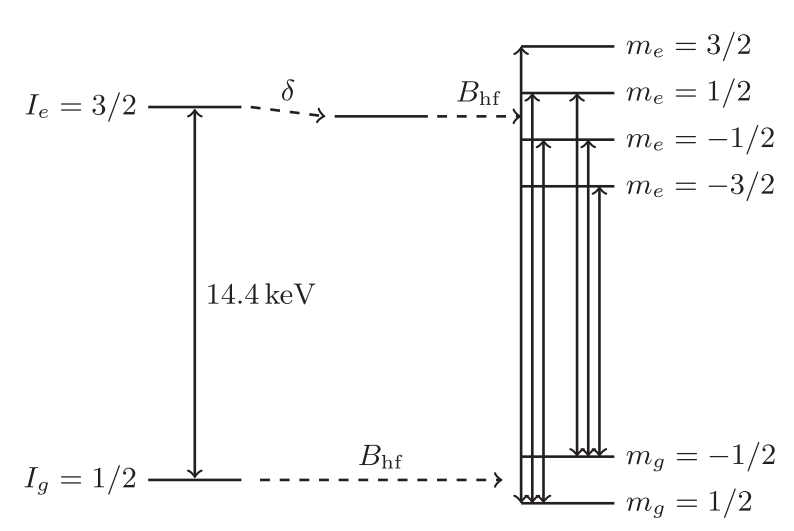

FIG. 4. Level scheme of ${ }^{57} \mathrm{Fe}$. The isomer shift $\delta$ shifts all excited states equally and is due to a monopole interaction with the local electric field. The addition of an external magnetic field $B_{\mathrm{hf}}$ results in a hyperfine splitting of the magnetic sublevels according to their spin projection $m_{g}$ or $m_{e}$ on the quantization axis. The six $M 1$ transitions are illustrated.

the thin layer of resonant nuclei is placed at the guided mode maximum. The low- $Z$ layer is thereby used as an inert filler which allows the precise positioning of the resonant layer at the desired depth in the cavity. The guided mode ensures the uniform illumination of all Mössbauer nuclei in the layer.

In most cases, the driven Mössbauer transitions present hyperfine splittings. Expressed in a multipole expansion, the most significant are the isomer shift, corresponding to the monopole interaction with the local electric field, the magnetic splitting due to a dipole interaction with the local magnetic field, and a quadrupole splitting resulting from the interaction with local electric-field gradients. The splittings are determined by the respective magnetic fields or electricfield gradients, which are in turn highly sensitive to the local electronic configuration. As most samples are polycrystalline, the hyperfine splittings will be inhomogeneous across the entire sample but homogeneous within an individual crystal domain. For the case of the 14.413-keV Mössbauer transition in ${ }^{57} \mathrm{Fe}$, the natural width is approximately $4.6 \mathrm{neV}$ and the transition has predominant magnetic dipole character. Magnetic splittings typically range in the interval $(1-50) \gamma_{0}$, while in iron carbides, for example, the isomer shift and quadrupole splitting range from $2 \gamma_{0}$ to $10 \gamma_{0}$ and from 0 to $0.55 \gamma_{0}$ [66], respectively, and vary for different crystal structures of the same chemical composition. An illustration of the isomer shift and magnetic splitting for ${ }^{57} \mathrm{Fe}$ is presented in Fig. 4.

The Mössbauer transition connects the ${ }^{57} \mathrm{Fe}$ ground state with spin $I_{g}=\frac{1}{2}$ with the first excited state with spin $I_{e}=$ $\frac{3}{2}$. The magnetic energy shift of each sublevel is given by the expression $m_{e(g)} \mu_{e(g)} B_{\mathrm{hf}}$, where $m_{e(g)}$ are the nuclear spin projections on the quantization axis, $\mu_{e(g)}$ is the magnetic moment of the nuclear excited (ground) states, and $B_{\mathrm{hf}}$ is the hyperfine magnetic field. For ${ }^{57} \mathrm{Fe}$, the excitedstate (ground-state) magnetic splittings amount to $3.26 \mathrm{neV} / \mathrm{T}$ $(5.71 \mathrm{neV} / \mathrm{T})$. Note that we have used electric dipole transitions in our general derivation, while the transitions in ${ }^{57} \mathrm{Fe}$ are magnetic dipole. This can be dealt with trivially by making a duality transformation [67], replacing the incident electric field $\mathbf{E}$ with the magnetic field $\mathbf{B}$, electric dipole moment $\wp \mathbf{d}$ with magnetic moment $\boldsymbol{\mu}$, and so forth. In particular, the electric dyadic Green's function is replaced with its magnetic dual

$$
\overleftrightarrow{G}\left(r, r^{\prime}\right) \rightarrow \overleftrightarrow{G}^{\star}\left(r, r^{\prime}\right)
$$

In the effective refractive index model of $x$-ray scattering in matter, the propagation is described via a frequencydependent index of refraction. Outside of resonant interactions, magnetic scattering is orders of magnitude weaker than electronic scattering [68]. Thus, the magnetic permeability of the layers can be taken to be that of the vacuum, $\mu_{0}$.

Therefore, in the notation of Buhmann [67], we can obtain the dual Green's tensor as

$$
\overleftrightarrow{G}^{\star}\left(r, r^{\prime}\right)=\frac{1}{\mu_{0}^{2}} \vec{\nabla} \times \overleftrightarrow{G}\left(r, r^{\prime}\right) \times \overleftarrow{\nabla}^{\prime}-\frac{1}{\mu_{0}} \overleftrightarrow{\delta}\left(r-r^{\prime}\right)
$$

The dyadic $\overleftrightarrow{\delta}$ term in the above transformation will modify each individual particle's Lamb shift and linewidth equally and as such can be absorbed into the definitions of $\omega_{0}$ and $\gamma_{0}$.

As the wavelengths of $\mathrm{x}$-ray photons are comparable to the lattice spacing of the resonant nuclei, in general the nuclei couple through a wide range of modes and this gives rise to the dynamical beating of nuclear forward scattering $[60,69]$. However, for grazing incidence geometry, it can nevertheless be shown that Fourier modes are approximately orthogonal (see Appendix A) and therefore we can make the single-mode approximation from before.

At grazing incidence, $s, p$ transmission and reflection are approximately equal and the $p$ polarization vector has negligible longitudinal component [70]. The relevant Fourier mode of the electric Green's function can therefore be approximated as a scalar times a projection matrix

$$
\overleftrightarrow{G}\left(\mathbf{k}_{0}, \omega_{0}\right) \approx \overleftrightarrow{\mathbb{1}}_{\perp} \mathcal{G}\left(\mathbf{k}_{0}, \omega_{0}\right)
$$

where $\overleftrightarrow{\mathbb{1}}_{\perp}=\overleftrightarrow{\mathbb{1}}-\hat{k}_{0} \otimes \hat{k}_{0}$. The same will apply to the magnetic propagation and therefore we can take the magnetic dual of this to be

$$
\overleftrightarrow{G}^{\star}\left(\mathbf{k}_{0}, \omega_{0}\right) \approx \overleftrightarrow{\mathbb{1}} \perp \frac{k_{0}^{2}}{\mu_{0}^{2}} \mathcal{G}\left(\mathbf{k}_{0}, \omega_{0}\right)
$$

This allows one to obtain the coherent and incoherent coupling strengths $J$ and $\Gamma$ as scalars directly from the Fourier transformed Green's function. In addition, the quantum optical approach of Heeg and Evers [64] demonstrates that these can also be understood in terms of the cavity detuning $\Delta_{C}$ and loss $\kappa$ of the cavity mode excited by the probe field such that

$$
J+i \Gamma \propto \frac{\Delta_{C}+i \kappa}{\Delta_{C}^{2}+\kappa^{2}} .
$$

In this model, the cavity detuning is minimized when the probe field is incident along one of the reflectivity minima of the cavity shown in Fig. 2(b) and increases when the angle is shifted away from the minimum. As such, in grazing incidence cavities, the coherent coupling constant $J$ is 
experimentally controllable by setting the angle of incidence of the probe field.

\section{A. Semiclassical versus quantum models for x-ray quantum optics with Mössbauer nuclei}

Before presenting some numerical examples, it is instructive to place the present formalism in the context of existing semiclassical and quantum models used in x-ray quantum optics. Previous approaches such as by Hannon et al. [71-75] as well as Sturhahn and Kohn [76,77] have modeled grazing incidence x-ray reflection using a diagrammatic expansion for the photonic scattering. The nuclear interaction is treated semiclassically, with the nuclear transitions taken to be linear dipole oscillators. In this approach, the results of Eq. (23) are implicitly modeled, but not explicitly obtainable. Specifically, the response of the nuclei is modeled according to Eq. (21) and the rescattering is implicitly included in the layer matrix formalism for the photonic propagation. However, this approach obscures the collective dynamics of the nuclei. In contrast, the present formalism makes the collective nuclear dynamics features explicit which allows both the coherent and incoherent effects to be investigated separately.

A more recent model, developed by Heeg and Evers [50,64], has focused on the quantum optical perspective, with the emphasis being on the resonant interaction of the nuclei with the cavity mode. A Green's-function approach for the scattering part of the Hamiltonian has been developed by Lentrodt et al. [51] and Kong et al. [56]. These works use analytic expressions for the Green's functions in layered media, developed by Tomaš [70] (and later on also by Johansson [78]), which can be expressed in terms of the layer matrix formalism for planar scattering. References [51,56] provide a connection between the linear response of the quantum optical model $[50,64]$ to the scattering models of Hannon et al. and Sturhahn and Kohn. Our work extends this quantum optical Green's-function approach to include inhomogeneous hyperfine parameters and provides insight into how the inhomogeneity will enter the nonlinear dynamics of the system. In the following we provide a few illustrative examples.

\section{B. Gaussian broadening for two-level systems}

As an illustrative example, we consider a layer of resonant Mössbauer nuclei with a Gaussian distribution of the isomer shift $\delta$. Such Gaussian distributions of hyperfine parameters are typical of amorphous solids [79-81]. For simplicity, we will consider the other hyperfine splittings to be negligible.

The isomer shift affects all excited states equally and the distribution does not affect the dipole vectors or the natural linewidths. As we are taking the other hyperfine splittings to be negligible, all states with a given nuclear spin $I$ are degenerate and we can model the system as having a single transition. Thus, we can treat the problem as scalar.

In the continuum limit, the coherent average becomes an integral over the distribution of $\delta$,

$$
\sum_{n} p_{n} \rightarrow \int_{-\infty}^{\infty} d \delta p(\delta)
$$

where $p(\delta)$ is the probability distribution finding a given value of $\delta$ in the ensemble. The response function $\mathcal{F}(\omega)$ is then

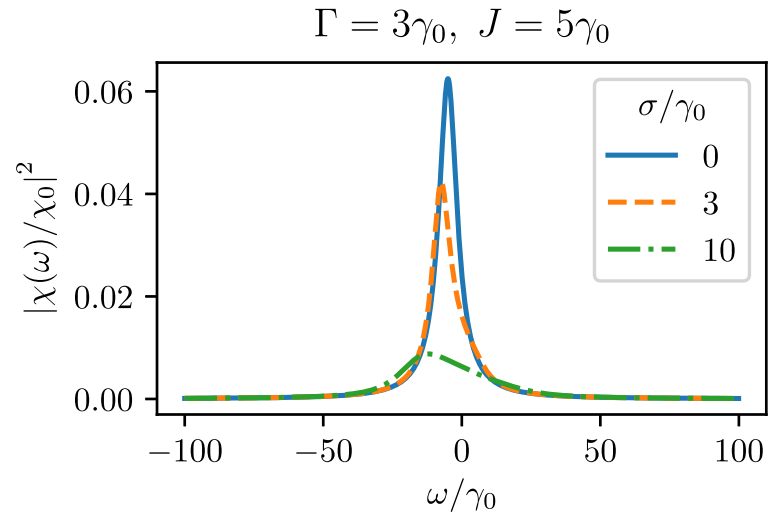

FIG. 5. Collective spectrum $\left|\chi(\omega) / \chi_{0}\right|^{2}$ as a function of the frequency $\omega$ for $J=5 \gamma_{0}$ and three different distribution widths $\sigma$. The peak is shifted further as the broadening is increased, and the shape is distorted.

given by

$$
\mathcal{F}(\omega)=\int d \delta p(\delta) \frac{\gamma_{0}}{\omega-\delta+i \gamma_{0}} .
$$

With a Gaussian distribution

$$
p(\delta ; \bar{\delta}, \sigma)=\frac{1}{\sqrt{2 \pi \sigma^{2}}} \exp \left(-\frac{(\delta-\bar{\delta})^{2}}{2 \sigma^{2}}\right),
$$

this evaluates to a Voigt profile [see Eq. (7.19) in [82]]

$$
\mathcal{F}(\omega)=\frac{\gamma_{0}}{\sqrt{2} \sigma} w\left(\frac{\omega-\bar{\delta}+i \gamma_{0}}{\sqrt{2} \sigma}\right),
$$

where

$$
w(z)=-i \sqrt{\pi} \exp \left(-z^{2}\right)[\operatorname{erfi}(z)-i] .
$$

As the dipole vectors are all along a single direction, we need only consider the component of susceptibility in this direction, given by

$$
\chi(\omega)=\chi_{0} \frac{\mathcal{F}(\omega)}{1+\gamma_{0}^{-1}(J+i \Gamma) \mathcal{F}(\omega)} .
$$

The interaction of the coherent collective coupling $J$ and the broadening $\sigma$ is particularly interesting. Unlike the case of purely collective broadening $\Gamma$, for significant distribution widths $\sigma$ the coherent coupling factor $J$ no longer acts as a simple Lamb shift. Indeed, if $J$ were to act as a Lamb shift, from (26) one would expect instead a susceptibility of

$$
\chi(\omega)=\chi_{0} F(\omega+J+i \Gamma),
$$

with a simple translation and broadening. Instead, the peak of the spectrum is shifted slightly further than it would be in the absence of the Gaussian broadening and is asymmetrically distorted. This is shown in Fig. 5, which presents the ratio $\left|\chi(\omega) / \chi_{0}\right|^{2}$ as a function of $\omega$ for three distributions widths $\sigma$.

At this point we find it interesting to compare our linear regime results with the work of Javanainen et al. $[36,37]$ and Jenkins et al. [38], who have examined the effect of inhomogeneous broadening on strongly coupled atomic clouds near saturation. In this regime, local spatial correlations become significant and permutation symmetry no longer applies. They 


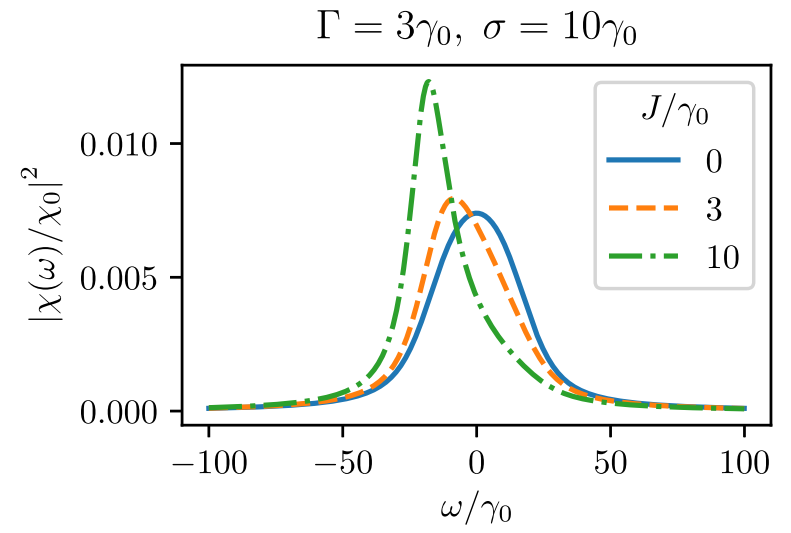

FIG. 6. Collective spectrum $\left|\chi(\omega) / \chi_{0}\right|^{2}$ as a function of the frequency $\omega$ for $\sigma=10 \gamma_{0}$ and three different collective coupling values $J$. With increasing coherent collective coupling $J$ the shape of the spectrum is distorted asymmetrically and the effective linewidth tends to $\Gamma$.

found that the local correlations in homogeneously broadened systems suppresses the Lorentz-Lorenz and collective Lamb shifts, while inhomogeneous broadening restores the superradiant mean-field dynamics [37]. We note that the case of inhomogeneous broadening shows some qualitative similarity to our linear regime result in Fig. 5, where we observe a larger peak shift for inhomogeneous broadening vs the unbroadened case.

For illustrative purposes, we have used values of $J$ and $\Gamma$ in the ranges (0-10) $\gamma_{0}$ and (3-5) $\gamma_{0}$, respectively, typical of $x$-ray cavities. For example, using PYNUSS to simulate the single line spectrum, we find that the cavity in Fig. 2 has $J=8.5 \gamma_{0}$ and $\Gamma=3.36 \gamma_{0}$ at an incident angle of $2.32 \mathrm{mrad}$, corresponding to just below the first reflection minimum. Going exactly to the first minimum gives $J=5.5 \gamma_{0}$ and increases $\Gamma$ significantly to $\Gamma=18.6 \gamma_{0}$. At an angle of $3.35 \mathrm{mrad}$, corresponding to the third reflection minimum, we have $J=1.79 \gamma_{0}$ and $\Gamma=$ $3.37 \gamma_{0}$. Typical hyperfine distribution widths are between 0 and $5 \gamma_{0}$. In optical contexts (for example, Doppler broadening in atomic clouds or size inhomogeneity in quantum dots), we would expect that the distribution widths could be substantially larger.

For significant distribution widths $\sigma \gg \gamma_{0}$, the line shape is that of a broad, almost Gaussian profile. However, as the collective coupling $J$ is increased, as well as being shifted and skewed, the effective linewidth tends to that of the incoherent coupling $\Gamma$. Figure 6 illustrates this behavior for increasing coherent collective coupling $J$.

\section{Interference effects for magnetic splitting}

Let us now consider the case of magnetic splitting in ${ }^{57} \mathrm{Fe}$ with no isomer shift and an x-ray field which drives the two $m_{e}-m_{g}=0$ transitions as shown in Fig. 4. Compared to the two-level system, the energies of these two transitions are now detuned by $\pm \phi= \pm \frac{1}{2}\left(\mu_{e}-\mu_{g}\right) B_{\mathrm{hf}}$. Our model Hamiltonian reads

$$
\begin{aligned}
H= & \hbar \phi\left(b_{1}^{\dagger} b_{1}-b_{2}^{\dagger} b_{2}\right)-\hbar J\left(b_{1}^{\dagger}+b_{2}^{\dagger}\right)\left(b_{1}+b_{2}\right) \\
& +\hbar \Omega\left(b_{1}^{\dagger}+b_{2}^{\dagger}\right)+\text { H.c },
\end{aligned}
$$

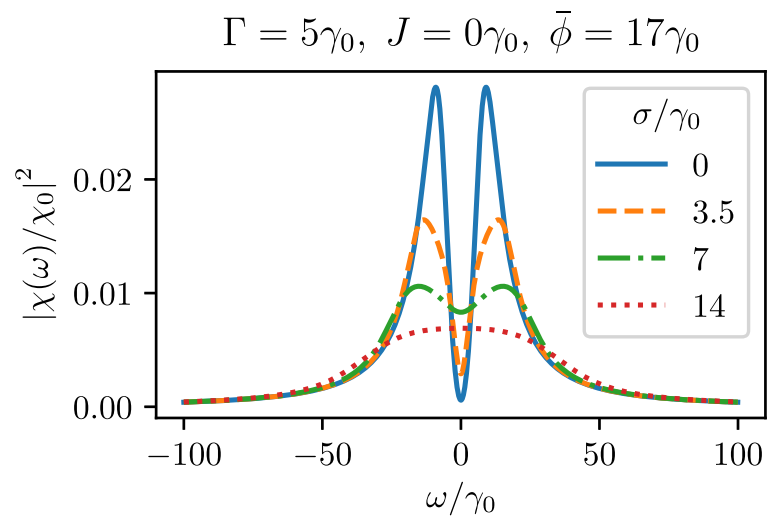

FIG. 7. Comparison of the collective spectrum $\left|\chi(\omega) / \chi_{0}\right|^{2}$ as a function of the frequency $\omega$ for $\Gamma=5 \gamma_{0}, J=0$, mean splitting $\bar{\phi}=17 \gamma_{0}$, and various values of the distribution width $\sigma$. As the distribution width increases, the dip is washed out to a very flat and broad peak.

with the Lindblad operator

$$
L[\rho]=-\gamma \sum_{i=1,2} \mathcal{L}\left[\rho, b_{i}^{\dagger}, b_{i}\right]-\Gamma \sum_{i, j=1,2} \mathcal{L}\left[\rho, b_{i}^{\dagger}, b_{j}\right] .
$$

The superradiant response of such a system was investigated by Kong and Pálffy [26] using an eigenvalue method. It was found that if the splitting $\phi$ is less than the incoherent part of the collective coupling $\Gamma$, the contributions from the two transitions interfere. The resulting spectrum has an interference dip in the peak, similar to electromagnetically induced transparency (EIT) [83], with the collective coupling $J$ playing the role of a control field. In addition, the coherent part of the collective coupling $J$ was found not to act as a simple Lamb shift but in fact nontrivially couple with the magnetic splitting, producing asymmetric Fano-like spectra.

We now consider this system with the addition of a Gaussian distribution of magnetic-field strengths across the sites. For a given site with splitting $\phi$, the response matrix is given by [26]

$$
\mathcal{F}(\omega ; \phi)=\frac{2 \gamma_{0}\left(\omega+i \gamma_{0}\right)}{\left(\omega+i \gamma_{0}\right)^{2}-\phi^{2}} .
$$

In the case of a completely uniform magnetic field, the collective susceptibility is therefore given by

$$
\chi(\omega)=\chi_{0} \frac{2 \gamma_{0}\left(\omega+i \gamma_{0}\right)}{\left(\omega+i \gamma_{0}\right)^{2}+2(J+i \Gamma)\left(\omega+i \gamma_{0}\right)-\phi^{2}} .
$$

This has two poles in the denominator,

$$
\omega_{ \pm}=-i \gamma_{0}-J-i \Gamma \pm \sqrt{(J+i \Gamma)^{2}+\phi^{2}} .
$$

When the collective coupling is completely incoherent $J=0$, the discriminant becomes $\sqrt{\phi^{2}-\Gamma^{2}}$. We can see that if $\phi<\Gamma$, the argument of the square root becomes negative and the poles become purely imaginary, describing overlapping Lorentzians with differing linewidths. This results in an EITlike dip. This behavior is illustrated in Fig. 7, which presents the susceptibility ratio $\left|\chi(\omega) / \chi_{0}\right|^{2}$ as a function of $\omega$ for four different values of the Gaussian distribution width $\sigma$. 


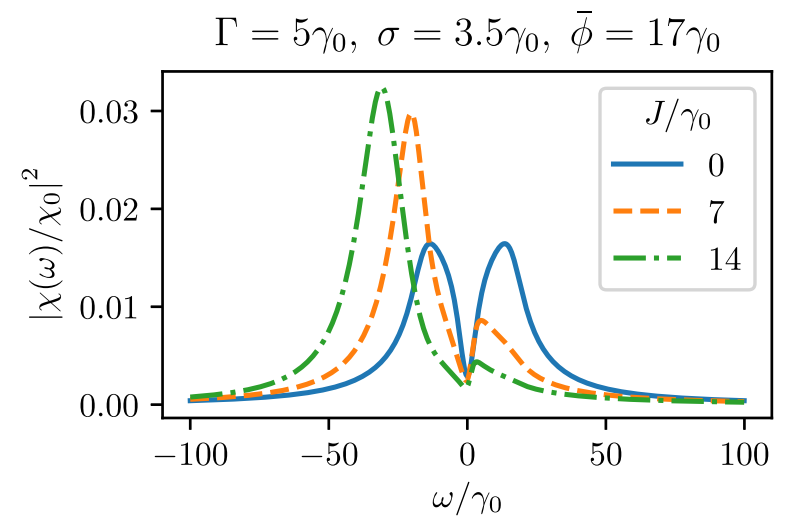

FIG. 8. Comparison of the collective spectrum $\left|\chi(\omega) / \chi_{0}\right|^{2}$ as a function of the frequency $\omega$ for small incoherent coupling $\Gamma=5 \gamma_{0}$, a small distribution width value $\sigma=3.5 \gamma_{0}$, mean splitting $\bar{\phi}=17 \gamma_{0}$, and varying values of the coherent collective coupling $J$.

If we now consider the magnetic splitting to have a Gaussian distribution of width $\sigma$ and mean $\bar{\phi}$, applying Eqs. (21) and (35) gives

$$
\begin{aligned}
\mathcal{F}(\omega) & =\int d \phi p(\phi ; \bar{\phi}, \sigma) F(\omega ; \phi) \\
& =\frac{\gamma_{0}}{\sqrt{2} \sigma}\left[w\left(\frac{\omega-\bar{\phi}+i \gamma_{0}}{\sqrt{2} \sigma}\right)+w\left(\frac{\omega+\bar{\phi}+i \gamma_{0}}{\sqrt{2} \sigma}\right)\right],
\end{aligned}
$$

with $w(z)$ given by Eq. (36). The susceptibility is as before given by

$$
\chi(\omega)=\chi_{0} \frac{\mathcal{F}(\omega)}{1+\gamma_{0}^{-1}(J+i \Gamma) \mathcal{F}(\omega)} .
$$

The overall envelope of the spectrum resembles that of the homogeneous case, and if the distribution width $\sigma$ is narrow compared with $\Gamma$ and $\bar{\phi}$, we can see that the intensity minimum is still resolvable. However, increasing the distribution width gradually flattens the dip and results in a flat broad peak as shown in Fig. 7.

More interesting is the effect of different strengths of the coherent collective coupling $J$. Rather than acting as a simple Lamb shift, the overall spectral shape is changed. One peak is flattened as the other increases, with large $J$ resulting in a completely asymmetric picture with only a single one of the contributions being resolved. This can be seen in Fig. 8, which presents the same dependence as Fig. 7 but this time for different values of $J$. While the peak locations are somewhat shifted, the shapes are distorted as well and the location of the minimum is unchanged. This is in contrast to the single line case, where $J$ acts as a pure Lamb shift.

This holds even when the distribution width is large enough that the minimum is not resolved, as shown in Fig. 9. For vanishing collective coupling $J=0$, the two peaks are merged and the effective linewidth is very broad. Increasing $J$ results in the left peak growing while the right peak decreases, and for significant $J$ only the left peak is individually resolved, with the linewidth approaching $2 \Gamma$. The result is an increase in the effective resolution of the spectrum, with an energy

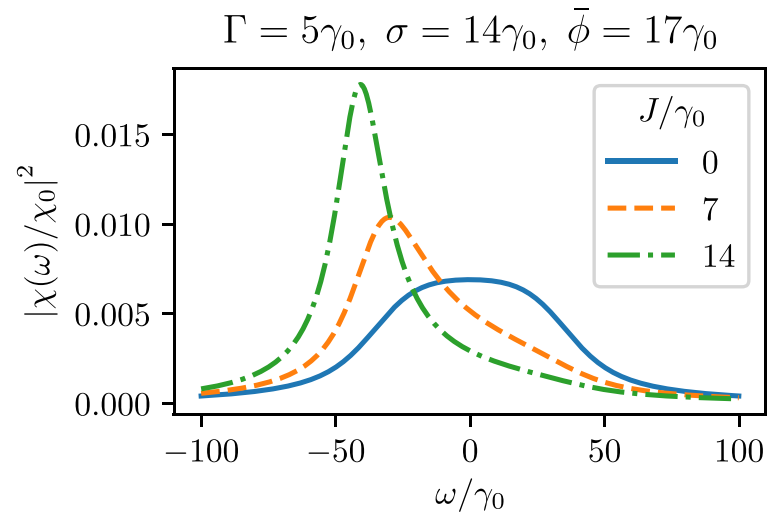

FIG. 9. Same as Fig. 8 but here for a wider distribution width $\sigma=14 \gamma_{0}$.

shift. As the coherent coupling strength is controlled via the angle of incidence of the driving field [51,64], this provides a mechanism for mechanical control of the linewidth of such a sample.

To understand this, we consider the matrix form of the corresponding equation of motion

$$
M\left(\begin{array}{l}
b_{1}(\omega) \\
b_{2}(\omega)
\end{array}\right)=\left(\begin{array}{l}
\Omega(\omega) \\
\Omega(\omega)
\end{array}\right)
$$

with

$$
M=(J+i \Gamma)\left(\begin{array}{ll}
1 & 1 \\
1 & 1
\end{array}\right)+\left(\begin{array}{cc}
\phi+i \gamma_{0} & 0 \\
0 & -\phi+i \gamma_{0}
\end{array}\right)
$$

and $\Omega(\omega)=\hbar^{-1} \wp E_{\mathrm{p}}(\omega)$. If $J$ is large enough compared with $\phi$ and $\gamma_{0}$, we may treat the second term as a small perturbation of the first. The eigenvectors of $M$ are then given by

$$
\hat{e}_{ \pm}=\frac{1}{\sqrt{2}}\left(\begin{array}{c}
1 \\
\pm 1
\end{array}\right)+O(\phi)
$$

with eigenvalues

$$
\lambda_{+}=2(J+i \Gamma)+O(\phi), \quad \lambda_{-}=O(\phi) .
$$

The driving term couples to $b_{1}$ and $b_{2}$ equally and is thus proportional to $\hat{e}_{+}$. Therefore, only the symmetric state $\hat{e}_{+}$is strongly driven and we will expect to see a single peak with a Lamb shift of $2 J$ and a broadening of $2 \Gamma$. If the collective broadening $\Gamma$ is significantly lower than the distribution width $\sigma$, we will then see a reduction in the effective linewidth. This has potential applications in samples with significant magnetic texture, with the beam angle of incidence on the sample being used to control the collective coupling and hence the effective linewidth.

\section{CONCLUSION}

In this paper we have examined an extension of the Dicke model for inhomogeneous atoms. We found a compact formula for the susceptibility in the weak-excitation regime, in terms of the coherently averaged nuclear or atomic responses, and the collective coupling constants $J$ and $\Gamma$. In addition to a collective Lamb shift and broadening, we found that the collective coupling also provides additional cross 
couplings between transitions, as well as spontaneously generated coherences.

Previous work by Kong and Pálffy [26] has shown that for homogeneous multilevel atoms, the collective coupling does not act as an overall broadening and Lamb shift and that in particular the coherent coupling $J$ distorts the shape of the line asymmetrically. We have shown that this conclusion holds in the case of inhomogeneous ensembles and that, in addition, the coherent coupling $J$ can counteract the inhomogeneous broadening.

In our model we have only considered linear dynamics and ignored the nonlinearity from local decay. The nonlinear dynamics of permutationally invariant systems, including local decay, have been well studied [23,25,59] and can be applied numerically to the subensembles. The Hilbert space of each subensemble scales with $N^{M+1}$, where $M$ is the number of transitions and $N$ is the maximum excitation number being modeled $[23,25]$. If the distribution of hyperfine parameters can be modeled in a piecewise constant fashion with $P$ pieces, then the Hilbert space can be modeled as the tensor product of $P$ subensembles, for a final dimension of $N^{P(M+1)}$. The scaling remains polynomial in excitation number but is exponential in subensemble number, which poses a considerable challenge for numerical simulation.

Our work is applicable to geometries that couple essentially via a single mode and are quasi-one-dimensional. For arbitrary geometries, our results hold if the driving field is weak and the scatterers are dense. This is the case for Mössbauer transitions in both nuclear forward geometry and in grazing incidence $\mathrm{x}$-ray cavities. Experiments with $\mathrm{X}$-ray scattering for both types of systems have shown that the Dicke model dynamics holds at currently experimentally accessible regimes $[13,14]$. However, going beyond the weak-excitation regime, the rescattered radiation will be emitted into different modes than the driving field and will therefore couple differently to the various transitions depending on the length and direction of travel. The problem then becomes highly geometrically dependent and the real-space Green's function must be used, with local spatial correlations taken into account [36-38,44,54,55,84].

\section{ACKNOWLEDGMENTS}

This work was funded by the Deutsches Forschungsgemeinschaft (DFG) through Projects No. 273811115 (SFB 1225 ISOQUANT) and No. 429529648 (TRR 306 QuCoLiMa) ("Quantum Cooperativity of Light and Matter"). A.P. acknowledges support from the Heisenberg Program of the DFG.

\section{APPENDIX A: SINGLE-MODE APPROXIMATION FOR POLARITON TRANSFORMATION}

In this Appendix we show that a coupling to Fourier modes other than the driving mode is negligible at low saturations. For simplicity, we consider only two-level systems; however, the argument can be adapted to multilevel systems by considering each pair of transition operators individually.

\section{Collective transition operators}

Let us define the collective raising and lowering operators $S_{ \pm}$and collective spin operator $Z$ as

$$
\begin{aligned}
Z(\mathbf{k}) & =\sum_{i} \sigma_{z}^{(i)} e^{i \mathbf{k} \cdot \mathbf{r}_{i}} \\
S_{ \pm}(\mathbf{k}) & =\sum_{i} \sigma_{ \pm}^{(i)} e^{i \mathbf{k} \cdot \mathbf{r}_{i}}
\end{aligned}
$$

They obey the commutation relations

$$
\begin{aligned}
{\left[S_{-}\left(\mathbf{k}_{1}\right), S_{+}\left(\mathbf{k}_{2}\right)\right] } & =-Z\left(\mathbf{k}_{1}+\mathbf{k}_{2}\right), \\
{\left[Z\left(\mathbf{k}_{1}\right), S_{ \pm}\left(\mathbf{k}_{2}\right)\right] } & = \pm 2 S_{ \pm}\left(\mathbf{k}_{1}+\mathbf{k}_{2}\right) .
\end{aligned}
$$

The operators can be centered on the driven wave vector $\mathbf{k}_{0}$ via the unitary transformation

$$
\left|e_{i}\right\rangle \rightarrow e^{-i \mathbf{k}_{0} \cdot \mathbf{r}_{i}}\left|e_{i}\right\rangle
$$

Under this transformation, we have

$$
\sigma_{+}^{(i)} \rightarrow \sigma_{+}^{(i)} e^{-i \mathbf{k}_{0} \cdot \mathbf{r}_{i}}, \quad \sigma_{z}^{(i)} \rightarrow \sigma_{z}^{(i)}
$$

and therefore

$$
Z(\mathbf{k}) \rightarrow Z(\mathbf{k}), \quad S_{ \pm}(\mathbf{k}) \rightarrow S_{ \pm}\left(\mathbf{k} \mp \mathbf{k}_{0}\right) .
$$

In particular, we note that $Z(0), S_{+}(\mathbf{k})$, and $S_{-}(-\mathbf{k})$ obey the usual $\mathrm{SU}(N)$ algebra of operators on symmetric Dicke states, and after the unitary transformation, $S_{ \pm}(0)$ are the collective transition operators of the driven mode.

\section{Orthogonality of Fourier modes}

The Fourier modes can be treated as orthogonal if the sum over atomic positions can be approximated as a $\delta$ function,

$$
\sum_{i} e^{i \mathbf{k} \cdot \mathbf{r}_{i}} \approx N \frac{(2 \pi)^{d}}{L^{d}} \delta^{d}(\mathbf{k}),
$$

where $\delta^{d}(\mathbf{k})$ is the Dirac delta function, $L$ is the length scale of the ensemble, and $d$ is the spatial dimension. We will consider this sum in three regimes: (a) dense samples, (b) dilute ordered lattices, and (c) dilute disordered lattices.

\section{a. Dense samples}

Equation (A6) can be seen to easily hold for dense ensembles such that the number density is much larger than the wavelength scale,

$$
\frac{N}{L^{d}} \lambda^{d} \ll 1 .
$$

In this case, one can approximate the sum over positions as an integral, which gives

$$
\sum_{i} e^{i \mathbf{k} \cdot \mathbf{r}_{i}} \approx \frac{N}{L^{d}} \int d^{d} r e^{i \mathbf{k} \cdot \mathbf{r}_{i}}=N \frac{(2 \pi)^{d}}{L^{d}} \delta^{d}(\mathbf{k}) .
$$

\section{b. Ordered lattices}

The case of crystalline samples has been extensively studied in condensed-matter physics. It is well known that even if the wavelength is comparable to the lattice spacing, for sufficiently large numbers of lattice sites the sum (A6) can 
be approximated by a sum over $\delta$ functions for the Bragg resonances of the lattice [85],

$$
\sum_{i} e^{i \mathbf{k} \cdot \mathbf{r}_{i}} \approx N \frac{(2 \pi)^{d}}{L^{d}} \sum_{\mathbf{K}} \delta^{d}(\mathbf{k}-\mathbf{K})
$$

where the $\mathbf{K}$ denote the reciprocal lattice vectors. If the lattice is placed in a waveguide, the Bragg scattered momenta which do not coincide with the direction of the guided mode will be rapidly decaying. As such, the contributions from the Bragg scattering can be modeled as an additional decay channel for the polariton.

\section{c. Disordered lattices}

Finally, Eq. (A6) also holds for any sufficiently large disordered sample, as the phase factors will be random and interfere destructively unless $\mathbf{k}$ is very close to zero. Indeed, for the particular case of uniformly distributed atoms, one can consider the sum to be a Monte Carlo integration of Eq. (A8), and so Eq. (A6) will hold for $N$ sufficiently large.

\section{Applicability to Mössbauer nuclei}

For Mössbauer samples we refer to Eq. (4.19) in [69] for a discussion of the width of the solid angle that the radiation is scattered to, which is proportional to

$$
\left|\sum_{i} e^{i\left(\mathbf{k}_{0}-\mathbf{k}\right) \cdot \mathbf{r}_{i}}\right|^{2}
$$

where $\mathbf{k}_{0}$ is the driving wave vector and $\mathbf{k}$ is the scattered wave vector. In particular, for the x-ray thin-film cavities the relevant estimate is for the two-dimensional crystalline case, which is applicable given our assumption of a very thin layer. This gives for the scattering solid angle

$$
\Delta \Omega \approx 6\left(\frac{\lambda^{2}}{A_{\|}}\right)^{3 / 4}
$$

where $A_{\|}$is the planar area of the cavity. In particular, since $\lambda$ is on the order of $1 \AA$ and the area on the order of $1 \mathrm{~mm}^{2}$, we find that the solid angle is on the order of $10^{-15} \mathrm{sr}$. As such, we see that Eq. (A6) is a good approximation in this regime. Even considering a sum over a single grain on the order of
$1 \mu \mathrm{m}^{2}$ in area, the solid angle is still very small, on the order of $10^{-6} \mathrm{sr}$.

In the case of perfectly crystalline samples, as discussed previously Bragg resonances must be considered. It is well known, however, that for grazing incidence, the reflection and transmission correspond to the (000) Bragg reflection, which preserves the planar wave vector [86].

\section{Action of lowering operators in symmetric subspace}

We consider the (nonnormalized) symmetric Dicke state with $m$ excitations,

$$
|m\rangle=S_{+}(0)^{m}|0\rangle .
$$

We consider the action of the collective lowering operator $S_{-}(\mathbf{k})$ on this state,

$$
S_{-}(\mathbf{k})|m\rangle=S_{-}(\mathbf{k}) S_{+}(0)^{m}|0\rangle .
$$

With the help of the commutation relations (A2), this leads to

$$
S_{-}(\mathbf{k})|m\rangle=\left[S_{+}(0) S_{-}(\mathbf{k})-Z(\mathbf{k})\right] S_{+}(0)^{m-1}|0\rangle .
$$

We can recursively expand this product and continue commuting $S_{-}(\mathbf{k})$ through to the right, until it annihilates the ground state. This then gives

$$
S_{-}(\mathbf{k})|m\rangle=-\sum_{l=0}^{m-1} S_{+}(0)^{l} Z(\mathbf{k}) S+(0)^{m-1-l}|0\rangle .
$$

We now consider

$$
\begin{aligned}
Z(\mathbf{k}) S_{+}(0)^{m}|0\rangle= & 2 S_{+}(\mathbf{k}) S_{+}(0)^{m}|0\rangle \\
& +S_{+}(0) Z(\mathbf{k}) S_{+}(0)^{m-1}|0\rangle .
\end{aligned}
$$

The base case is

$$
Z(\mathbf{k})|0\rangle=-\sum_{i} e^{i \mathbf{k} \cdot \mathbf{r}_{i}}|0\rangle \approx-N \frac{(2 \pi)^{d}}{L^{d}} \delta^{d}(\mathbf{k})|0\rangle .
$$

By recursion, we can see that

$$
Z(\mathbf{k}) S_{+}(0)^{m}|0\rangle=2 m S_{+}(\mathbf{k}) S_{+}(0)^{m-1}|0\rangle-N \frac{(2 \pi)^{d}}{L^{d}} S_{+}(0)^{m}|0\rangle .
$$

This finally gives

$$
\begin{aligned}
S_{-}(\mathbf{k})|m\rangle & =-\sum_{l=0}^{m-1} 2(m-l-1) S_{+}(\mathbf{k}) S_{+}^{m-2}|0\rangle-N \frac{(2 \pi)^{d}}{L^{d}} \delta^{d}(\mathbf{k}) S_{+}(0)^{m-1}|0\rangle \\
& =m\left(N \frac{(2 \pi)^{d}}{L^{d}} \delta^{d}(\mathbf{k}) S_{+}(0)-(m-1) S_{+}(\mathbf{k})\right)|m-2\rangle .
\end{aligned}
$$

In particular, we see that the second term involving the nonsymmetric state $S_{+}(\mathbf{k})|m-2\rangle$ is smaller than the symmetric state by a factor of $(m-1) / N$. At low saturations, this is negligible. Therefore, if the state is driven to a symmetric state with wave vector $\mathbf{k}_{0}$, the Hamiltonian for low excitations has a sharp resonance in the symmetric subspace and we can make a single-mode approximation. In particular, since we can express a translationally symmetric Green's function in the form

$$
\overleftrightarrow{G}\left(\mathbf{r}_{1}, \mathbf{r}_{2}, \omega_{0}\right)=\int \frac{L^{d} d^{d} k}{(2 \pi)^{d}} \overleftrightarrow{\mathcal{G}}\left(\mathbf{k}, \omega_{0}\right) e^{i \mathbf{k} \cdot\left(\mathbf{r}_{1}-\mathbf{r}_{2}\right)},
$$

the single-mode dynamics allows us to make the replacement

$$
\overleftrightarrow{G}\left(\mathbf{r}_{1}, \mathbf{r}_{2}, \omega_{0}\right) \rightarrow \overleftrightarrow{\mathcal{G}}\left(\mathbf{k}_{0}, \omega_{0}\right) e^{i \mathbf{k}_{0} \cdot\left(\mathbf{r}_{1}-\mathbf{r}_{2}\right)}
$$




\section{APPENDIX B: APPROXIMATING SINGLE-PARTICLE DECAY AS COLLECTIVE}

To justify approximating the single-particle decay as collective, we note that we can write the exact form of $L_{\mathrm{A}}$ as

$$
\begin{aligned}
L_{A}[\rho] & =-\sum_{\mu, i} \hbar \gamma_{\mu}\left(\left|e_{\mu}^{(i)}\right\rangle\left\langle e_{\mu}^{(i)}|\rho+\rho| e_{\mu}^{(i)}\right\rangle\left\langle e_{\mu}^{(i)}|-2| g_{\mu}^{(i)}\right\rangle\left\langle e_{\mu}^{(i)}|\rho| e_{\mu}^{(i)}\right\rangle\left\langle g_{\mu}^{(i)}\right|\right) \\
& =-\sum_{\mu} \hbar \gamma_{\mu}\left(b_{\mu}^{\dagger} b_{\mu} \rho+\rho b_{\mu}^{\dagger} b_{\mu}+\sum_{i}\left|g_{\mu}^{(i)}\right\rangle\left\langle g_{\mu}^{(i)}\right|\left\langle e_{\mu}^{(i)}|\rho| e_{\mu}^{(i)}\right\rangle\right) .
\end{aligned}
$$

At low saturations $\left\langle e_{\mu}^{(i)}|\rho| e_{\mu}^{(i)}\right\rangle \approx 0$; therefore, both the single-particle and collective decays give the same result,

$$
L_{A}[\rho] \approx-\sum_{\mu} \hbar \gamma_{\mu}\left\{b_{\mu}^{\dagger} b_{\mu}, \rho\right\} .
$$

In addition, for X-ray quantum optics the cavities considered are in the so-called bad cavity regime and the cavity mode lifetime is much shorter than the natural lifetime of the resonant transitions. Therefore, the incoherent processes are dominated by collective interactions and the internal decay can be approximated as a small correction to the collective decay rates.

\section{APPENDIX C: MULTIPLE GROUND STATES}

We now consider an atom with multiple ground states as well as multiple excited states. As with the excited states, the ground states are considered to have small splittings compared with the energy difference between the ground and excited bands, and dipole transitions are forbidden between ground states. We index here the excited states with greek indices $\mu, v \ldots$ and the ground states with latin indices $j, k \ldots$

For nuclei with multiple ground states, we further partition the ensembles by the initial ground states of the atoms, creating permutationally invariant subensembles. For a given subensemble, we use the initial ground state $g_{0}$ as the "vacuum" state for the generalized Holstein-Primakoff transformation. We then obtain

$$
\begin{aligned}
\sum_{i=1}^{N}\left|e_{\mu}^{(i)}\right\rangle\left\langle g_{0}^{(i)}\right| & \approx \sqrt{N} b_{\mu}^{\dagger}, \\
\sum_{i=1}^{N}\left|g_{j}^{(i)}\right\rangle\left\langle g_{0}^{(i)}\right| & \approx \sqrt{N} c_{j}^{\dagger}, \\
\sum_{i=1}^{N}\left|g_{j}^{(i)}\right\rangle\left\langle g_{k}^{(i)}\right| & =c_{j}^{\dagger} c_{k}, \\
\sum_{i=1}^{N}\left|e_{\mu}^{(i)}\right\rangle\left\langle g_{j}^{(i)}\right| & =b_{\mu}^{\dagger} c_{j}, \\
\sum_{i=1}^{N}\left|e_{\mu}^{(i)}\right\rangle\left\langle e_{\nu}^{(i)}\right| & =b_{\mu}^{\dagger} b_{\nu}, \\
{\left[b_{\mu}, b_{\nu}^{\dagger}\right] } & =\delta_{\mu \nu}, \\
{\left[c_{j}, c_{k}^{\dagger}\right] } & =\delta_{j k}, \\
{\left[c_{j}, b_{\mu}^{\dagger}\right] } & =0 .
\end{aligned}
$$

The single-particle Hamiltonian then reads

$$
H_{\mathrm{A}}=\sum_{\mu}\left(\Delta_{\mu}-\delta_{0}\right) b_{\mu}^{\dagger} b_{\mu}+\sum_{j}\left(\delta_{j}-\delta_{0}\right) c_{j}^{\dagger} c_{j},
$$

with the single-particle decay reading

$$
L_{\mathrm{A}}[\rho]=-\sum_{\mu} \gamma_{\mu 0} \mathcal{L}\left[\rho, b_{\mu}^{\dagger}, b_{\mu}\right]-\sum_{\mu, j} \gamma_{\mu j} \mathcal{L}\left[\rho, b_{\mu}^{\dagger} c_{j}, c_{j}^{\dagger} b_{\mu}\right]
$$

where $\gamma_{\mu 0}$ is the decay rate to the initial ground state and $\gamma_{\mu j}$ is the decay rate to ground state $j$. If we consider the action of this superoperator on $b_{\mu}$, we obtain

$$
L_{\mathrm{A}}\left[b_{\mu}\right]=-\gamma_{\mu 0} b_{\mu}-\sum_{j} \gamma_{\mu_{j}}\left(1+c_{j}^{\dagger} c_{j}\right) b_{\mu} .
$$

The action on a ground-state operator reads

$$
L_{A}\left[c_{j}\right]=\sum_{\mu} \gamma_{\mu j} b_{\mu}^{\dagger} b_{\mu} c_{j}
$$

The rate of population transfer to the other ground state is thus proportional to the excited-state populations, which are negligible in the linear-response regime. Therefore, we can assume $c_{j} c_{k} \approx 0$ for all $j$ and $k$. However, the decay rate of the transition operator $b_{\mu}$ is still affected by the leftover terms,

$$
L_{\mathrm{A}}\left[b_{\mu}\right] \approx-\left(\gamma_{\mu 0}+\sum_{j} \gamma_{\mu j}\right) b_{\mu}=-\gamma_{\mu} b_{\mu}
$$

i.e., the effective decay rate $\gamma_{\mu}$ for a transition operator $b_{\mu}$ is the sum of the decay rates of all decay channels for excited state $\mu$. Thus, we may write

$$
L_{\mathrm{A}}[\rho] \approx-\sum_{\mu} \gamma_{\mu} \mathcal{L}\left[\rho, b_{\mu}^{\dagger}, b_{\mu}\right]
$$

In addition to the internal decay, the cavity mediated coupling includes transitions to different ground states than the initial. The interaction Hamiltonian reads

$$
\begin{aligned}
H_{\mathrm{dd}}= & -J \sum_{\mu, \nu} \mathbf{d}_{\mu 0}^{\perp} \cdot \mathbf{d}_{\nu 0}^{* \perp} b_{\mu}^{\dagger} b_{v} \\
& -\frac{J}{\sqrt{N}} \sum_{\mu, v} \sum_{m} \mathbf{d}_{\mu 0}^{\perp} \cdot \mathbf{d}_{\nu j}^{* \perp} b_{\mu}^{\dagger} b_{\nu} c_{j}^{\dagger}+\text { H.c. } \\
& -\frac{J}{N} \sum_{\mu, v} \sum_{j, k} b_{\mu}^{\dagger} b_{\nu} c_{j}^{\dagger} c_{k} .
\end{aligned}
$$


We can see that terms involving population transfer between ground states are suppressed by a factor of $\sqrt{N}$ or higher and thus can be neglected in the linear-response regime. An analogous argument holds for the dipole-dipole Lindblad term and for the probe field driving. Thus, we conclude that for the linear response in the presence of multiple ground states, only the initial ground state of a subensemble needs to be considered, with the only contributions of the other ground states being to the decay rates of the transition operators.
[1] R. H. Dicke, Phys. Rev. 93, 99 (1954).

[2] N. E. Rehler and J. H. Eberly, Phys. Rev. A 3, 1735 (1971).

[3] M. Gross and S. Haroche, Phys. Rep. 93, 301 (1982).

[4] C. Leonardi, F. Persico, and G. Vetri, Riv. Nuovo Cimento 9, 1 (1986).

[5] N. Skribanowitz, I. P. Herman, J. C. MacGillivray, and M. S. Feld, Phys. Rev. Lett. 30, 309 (1973).

[6] S. Inouye, A. P. Chikkatur, D. M. Stamper-Kurn, J. Stenger, D. E. Pritchard, and W. Ketterle, Science 285, 571 (1999).

[7] K. Baumann, C. Guerlin, F. Brennecke, and T. Esslinger, Nature (London) 464, 1301 (2010).

[8] M. Scheibner, T. Schmidt, L. Worschech, A. Forchel, G. Bacher, T. Passow, and D. Hommel, Nat. Phys. 3, 106 (2007).

[9] A. Angerer, K. Streltsov, T. Astner, S. Putz, H. Sumiya, S. Onoda, J. Isoya, W. J. Munro, K. Nemoto, K. Schiedmeyer, and J. Majer, Nat. Phys. 14, 1168 (2018).

[10] M. O. Araújo, I. Krešić, R. Kaiser, and W. Guerin, Phys. Rev. Lett. 117, 073002 (2016).

[11] F. Cottier, R. Kaiser, and R. Bachelard, Phys. Rev. A 98, 013622 (2018).

[12] B. W. Adams, C. Buth, S. M. Cavaletto, J. Evers, Z. Harman, C. H. Keitel, A. Pálffy, A. Picón, R. Röhlsberger, Y. Rostovtsev, and K. Tamasaku, J. Mod. Opt. 60, 2 (2013).

[13] R. Röhlsberger, K. Schlage, B. Sahoo, S. Couet, and R. Rüffer, Science 328, 1248 (2010).

[14] A. I. Chumakov, A. Q. R. Baron, I. Sergueev, C. Strohm, O. Leupold, Y. Shvyd'ko, G. V. Smirnov, R. Rüffer, Y. Inubushi, M. Yabashi, K. Tono, T. Kudo, and T. Ishikawa, Nat. Phys. 14, 261 (2017).

[15] R. Reimann, W. Alt, T. Kampschulte, T. Macha, L. Ratschbacher, N. Thau, S. Yoon, and D. Meschede, Phys. Rev. Lett. 114, 023601 (2015).

[16] J. Kim, D. Yang, S.-h. Oh, and K. An, Science 359, 662 (2018).

[17] M. O. Scully, E. S. Fry, C. H. R. Ooi, and K. Wódkiewicz, Phys. Rev. Lett. 96, 010501 (2006).

[18] R. A. de Oliveira, M. S. Mendes, W. S. Martins, P. L. Saldanha, J. W. R. Tabosa, and D. Felinto, Phys. Rev. A 90, 023848 (2014).

[19] F. Rajabi and M. Houde, Astrophys. J. 826, 216 (2016).

[20] C. Vieira, M. Pardal, J. T. Mendonća, and A. R. Fonseca, Nat. Phys. 17, 99 (2021).

[21] P. T. Arecchi, E. Gilmore, and D. M. Kim, Lett. Nuovo Cimento 6, 219 (1973).

[22] C. A. Müller, C. Miniatura, D. Wilkowski, R. Kaiser, and D. Delande, Phys. Rev. A 72, 053405 (2005).

[23] M. Gegg and M. Richter, New J. Phys. 18, 043037 (2016).

[24] R. T. Sutherland and F. Robicheaux, Phys. Rev. A 95, 033839 (2017).

[25] M. Gegg, Identical emitters, collective effects and dissipation in quantum optics, Ph.D. thesis, Technische Universität Berlin, 2017.
[26] X. Kong and A. Pálffy, Phys. Rev. A 96, 033819 (2017).

[27] S. Das, V. E. Elfving, F. Reiter, and A. S. Sørensen, Phys. Rev. A 97, 043837 (2018).

[28] G. S. Agarwal, Phys. Rev. A 4, 1791 (1971).

[29] J. H. Eberly, Lett. Nuovo Cimento 1, 182 (1971).

[30] J. H. Eberly, Acta Phys. Pol. A 39, 633 (1971).

[31] R. Jodoin and L. Mandel, Phys. Rev. A 9, 873 (1974).

[32] C. Leonardi and A. Vaglica, Nuovo Cimento B 67, 256 (1982).

[33] F. C. Spano and S. Mukamel, J. Chem. Phys. 91, 683 (1989).

[34] V. V. Temnov and U. Woggon, Phys. Rev. Lett. 95, 243602 (2005).

[35] S. B. Jäger, H. Liu, A. Shankar, J. Cooper, and M. J. Holland, Phys. Rev. A 103, 013720 (2021).

[36] J. Javanainen, J. Ruostekoski, Y. Li, and S.-M. Yoo, Phys. Rev. A 96, 033835 (2017).

[37] J. Javanainen, J. Ruostekoski, Y. Li, and S.-M. Yoo, Phys. Rev. Lett. 112, 113603 (2014).

[38] S. D. Jenkins, J. Ruostekoski, J. Javanainen, R. Bourgain, S. Jennewein, Y. R. P. Sortais, and A. Browaeys, Phys. Rev. Lett. 116, 183601 (2016).

[39] A. S. Kuraptsev and I. M. Sokolov, Phys. Rev. A 94, 022511 (2016).

[40] R. Bonifacio and L. A. Lugiato, Phys. Rev. A 11, 1507 (1975).

[41] F. Haake, J. Haus, H. King, G. Schröder, and R. Glauber, Phys. Rev. Lett. 45, 558 (1980).

[42] A. Ishikawa, K. Miyajima, M. Ashida, T. Itoh, and H. Ishihara, J. Phys. Soc. Jpn. 85, 034703 (2016).

[43] S. J. Roof, K. J. Kemp, M. D. Havey, and I. M. Sokolov, Phys. Rev. Lett. 117, 073003 (2016).

[44] J. Ruostekoski and J. Javanainen, Phys. Rev. Lett. 117, 143602 (2016).

[45] A. Asenjo-Garcia, J. D. Hood, D. E. Chang, and H. J. Kimble, Phys. Rev. A 95, 033818 (2017).

[46] S. Das, V. E. Elfving, F. Reiter, and A. S. Sørensen, Phys. Rev. A 97, 043838 (2018).

[47] M. G. Benedict and E. D. Trifonov, Phys. Rev. A 38, 2854 (1988).

[48] A. M. Samson, Y. A. Logvin, and S. I. Turovets, Opt. Commun. 78, 208 (1990).

[49] S.-H. Lim, T. G. Bjorklund, F. C. Spano, and C. J. Bardeen, Phys. Rev. Lett. 92, 107402 (2004).

[50] K. P. Heeg and J. Evers, Phys. Rev. A 91, 063803 (2015).

[51] D. Lentrodt, K. P. Heeg, C. H. Keitel, and J. Evers, Phys. Rev. Res. 2, 023396 (2020).

[52] T. Gruner and D.-G. Welsch, Phys. Rev. A 53, 1818 (1996).

[53] H. T. Dung, L. Knöll, and D.-G. Welsch, Phys. Rev. A 66, 063810 (2002).

[54] A. Svidzinsky and J.-T. Chang, Phys. Rev. A 77, 043833 (2008).

[55] A. A. Svidzinsky, J.-T. Chang, and M. O. Scully, Phys. Rev. A 81, 053821 (2010). 
[56] X. Kong, D. E. Chang, and A. Pálffy, Phys. Rev. A 102, 033710 (2020).

[57] M. Wubs, L. G. Suttorp, and A. Lagendijk, Phys. Rev. A 70, 053823 (2004).

[58] Z. Kurucz and K. Mølmer, Phys. Rev. A 81, 032314 (2010).

[59] N. Shammah, S. Ahmed, N. Lambert, S. De Liberato, and F. Nori, Phys. Rev. A 98, 063815 (2018).

[60] R. Röhlsberger, Nuclear Condensed Matter Physics with Synchrotron Radiation: Basic Principles, Methodology and Applications (Springer Science+Business Media, New York, 2004).

[61] J. P. Hannon, G. T. Trammell, M. Blume, and D. Gibbs, Phys. Rev. Lett. 61, 1245 (1988).

[62] M. O. Scully and A. A. Svidzinsky, Science 328, 1239 (2010).

[63] P. Y. Wen, K.-T. Lin, A. F. Kockum, B. Suri, H. Ian, J. C. Chen, S. Y. Mao, C. C. Chiu, P. Delsing, F. Nori, G.-D. Lin, and I.-C. Hoi, Phys. Rev. Lett. 123, 233602 (2019).

[64] K. P. Heeg and J. Evers, Phys. Rev. A 88, 043828 (2013).

[65] K. P. Heeg (private communication).

[66] X.-W. Liu, S. Zhao, Y. Meng, Q. Peng, A. K. Dearden, C.-F. Huo, Y. Yang, Y.-W. Li, and X.-D. Wen, Sci. Rep. 6, 26184 (2016).

[67] S. Y. Buhmann, Dispersion Forces I: Macroscopic Quantum Electrodynamics and Ground-State Casimir, Casimir-Polder and van der Waals Forces, Springer Tracts in Modern Physics Vol. 247 (Springer, Berlin, 2012), Sec. 2.1.4.

[68] J. D. Jackson, Classical Electrodynamics (Wiley, New York, 1999).
[69] J. Hannon and G. Trammell, Hyperfine Interact. 123/124, 127 (1999).

[70] M. S. Tomaš, Phys. Rev. A 51, 2545 (1995).

[71] J. P. Hannon and G. T. Trammell, Phys. Rev. 186, 306 (1969).

[72] J. P. Hannon, N. J. Carron, and G. T. Trammell, Phys. Rev. B 9 , 2791 (1974).

[73] J. P. Hannon and G. T. Trammell, Phys. Rev. 169, 315 (1968).

[74] J. P. Hannon, G. T. Trammell, M. Mueller, E. Gerdau, R. Rüffer, and H. Winkler, Phys. Rev. B 32, 6363 (1985).

[75] J. P. Hannon, G. T. Trammell, M. Mueller, E. Gerdau, R. Rüffer, and H. Winkler, Phys. Rev. B 32, 6374 (1985).

[76] W. Sturhahn, J. Phys.: Condens. Matter 16, S497 (2004).

[77] W. Sturhahn and V. Kohn, Hyperfine Interact. 123/124, 367 (1999).

[78] P. Johansson, Phys. Rev. B 83, 195408 (2011).

[79] T. E. Sharon and C. C. Tsuei, Phys. Rev. B 5, 1047 (1972).

[80] A. M. van Diepen and T. J. A. Popma, J. Phys. (Paris) Colloq. 37, C6-755 (1976).

[81] G. Férey, A. Leclerc, R. de Pape, J. Mariot, and F. Varret, Solid State Commun. 29, 477 (1979).

[82] NIST Digital Library of Mathematical Functions, version 1.0.28, http://dlmf.nist.gov/.

[83] M. Fleischhauer, A. Imamoglu, and J. P. Marangos, Rev. Mod. Phys. 77, 633 (2005).

[84] E. Ressayre and A. Tallet, Phys. Rev. A 15, 2410 (1977).

[85] M. P. Marder, in Condensed Matter Physics (Wiley, Hoboken, 2010), pp. 897-902.

[86] R. Röhlsberger, Hyperfine Interact. 123/124, 455 (1999). 